\title{
Resolvendo conflitos em aplicações de distribuição de conteúdo em redes veiculares
}

\author{
Ronan D. Mendonça ${ }^{1,2}$, Thais R. M. Braga Silva ${ }^{2}$, Fabrício A. Silva ${ }^{2}$, Linnyer B. Ruiz ${ }^{1,3}$ \\ ${ }^{1}$ Programa de Pós-Graduação em Engenharia Elétrica \\ Universidade Federal de Minas Gerais (UFMG), MG - Brasil \\ ${ }^{2}$ Instituto de Ciências Exatas e Tecnológicas \\ Universidade Federal de Viçosa (UFV) - Campus Florestal, MG - Brasil \\ ${ }^{3}$ Departamento de informática \\ Universidade Estadual de Maringá (UEM) - Maringá , PR - Brasil \\ \{ronan.dutra, thais.braga, fabricio.asilva\}@ufv.br, linnyer@gmail.com
}

\begin{abstract}
The distribution of entertainment content in vehicular networks consume a large volume of bandwidth. When solicitations are not attended, it occurs conflicts caused by the dispute for resources, reducing the users' satisfaction. This paper proposes a solution to solve these conflicts, considering the best algorithm according to environmental contexts and the application, through the CReMe methodology. The obtained results show that was possible use the methodology to solve those conflicts, and still be adaptative to the context, balancing the use of resources and the users' satisfaction.
\end{abstract}

Resumo. A distribuição de conteúdo de entretenimento em redes veiculares consome um volume significativo de largura de banda. Quando as solicitações de conteúdos não são totalmente atendidas, ocorrem conflitos de interesses na disputa pelos recursos, reduzindo a satisfação dos usuários. Este trabalho propõe uma solução para resolver estes conflitos, considerando o algoritmo mais adequado ao contexto do ambiente e da aplicação, por meio da metodologia CReMe. Os resultados obtidos mostraram que foi possível utilizar a metodologia para resolver os conflitos, de modo adaptativo ao contexto, balanceando o consumo de recursos e a satisfação do usuário.

\section{Introdução}

As redes veiculares têm como função viabilizar uma conexão para a troca de mensagens e compartilhamento de recursos entre os próprios veículos e ou entre os veículos e estações fixas. Aplicações de entretenimento para as redes veiculares têm a função de distribuir conteúdos como músicas, vídeos, páginas web e são de grande importância e utilidade por atuarem em um ambiente cada vez mais caótico, implicado por problemas gerados pelo próprio trânsito dos veículos [Costa-Montenegro et al. 2012]. Um dos grandes desafios inerentes às aplicações de entretenimento é a alta demanda por largura de banda [Jiau et al. 2015].

Para as aplicações de uso coletivo disponibilizarem seus recursos, com todos os seus benefícios, são imprescindíveis a utilização dos dados de contexto dos usuários e o ambiente. Os dados de contexto são informações relevantes ao sistema e referem à 
situação em que se encontra o ambiente e o próprio usuário [Perera et al. 2014]. As aplicações veiculares cientes de contexto são também coletivas, uma vez que os recursos utilizados são compartilhados por vários veículos simultaneamente. No instante, vários dos recursos disponibilizados pelas aplicações são limitados e podem se tornar escassos, comprometendo a utilização da aplicação e gerando uma disputa coletiva por estes recursos [Jiau et al. 2015]. Para não deixar que os recursos se esgotem e os usuários fiquem completamente insatisfeitos, torna-se necessário a detecção e o tratamento dos conflitos ocorridos por metodologias e procedimentos específicos ao problema.

O objetivo deste trabalho é propor uma solução para identificar e tratar conflitos em aplicações de entretenimento para redes veiculares. Para isso, será utilizada a metodologia CReMe (Conflict Resolution Methodology) [Silva. 2010], proposta para resolver conflitos em sistemas coletivos, ubíquos e cientes de contexto. Foram agregados a esta metodologia, as particularidades das redes veiculares e algoritmos específicos para a resolução dos conflitos pertinentes ao ambiente veicular. Por meio desta metodologia, espera-se que o consumo de recursos seja balanceado com a satisfação dos usuários, fazendo com que os usuários sejam atendidos sem que os recursos sejam degradados.

As próximas seções deste artigo estão organizadas da seguinte forma: na Seção 2 são apresentados os trabalhos relacionados; na Seção 3 é apresentada a solução proposta por este trabalho; na Seção 4 são descritos os detalhes da simulação e apresentados os resultados obtidos juntamente com as análises dos mesmos; e a Seção 5 apresenta as considerações finais e os trabalhos futuros.

\section{Trabalhos relacionados}

A discussão dos trabalhos relacionados abrange os estudos que envolvem conteúdo de entretenimento, utilização de dados de contexto e taxa de transmissão em redes veiculares.

Com relação à entrega de conteúdo de entretenimento, o trabalho de [Sarakis et al. 2016] propõe uma avaliação da capacidade das redes veiculares na transmissão de conteúdos multimídia. A avaliação incide na camada de aplicação para streaming de vídeos. Os autores realizaram testes para diferentes tipos de vídeos, velocidades e ambientes, concluindo que uma qualidade aceitável de transmissão de conteúdos multimídia pode ser alcançada em redes veiculares. A abordagem proposta por [Yang et al. 2012] tem como objetivo principal a codificação de dados para distribuição de fluxo contínuo de conteúdo multimídia em redes veiculares. Os autores relatam o problema de se transmitir conteúdo de entretenimento em redes veiculares com baixa largura de banda disponível. Propuseram então, um algoritmo de escalonamento de transmissão oportunista para melhorar o desempenho em diferentes densidades da rede. [Jiau et al. 2015] apresentam e avaliam arquiteturas para serviços multimídia em redes veiculares baseadas em nuvens. Por meio de uma comparação da estabilidade, capacidade de extensão, gestão e outras características, os autores concluem que a forma híbrida, onde um veículo faz o papel da infraestrutura e de um usuário final ao mesmo tempo, é a mais indicada para utilização de serviços multimídia.

Com relação aos dados contextuais, existem trabalhos que focam na utilização destes para uma distribuição eficiente de recursos. Em [Wan et al. 2014], os autores apresentam um proposta de arquitetura em camadas baseada em dados de contexto para utilização de redes sociais e aplicações de segurança em redes veiculares. As camadas têm 
como objetivos compartilhar conexão e recursos de informação de tráfego e de entretenimento aos veículos, fornecer informações de contexto e interação dos serviços disponíveis e disponibilizar aplicações e serviços em nuvem. [Sepulcre et al. 2015] propõe uma técnica de uso de informações de contexto para decidir qual tecnologia de comunicação é mais adequada ao uso em cada momento. As decisões são feitas diretamente por cada veículo com a assistência da infraestrutura na aquisição dos dados de contexto. Os autores apresentam os resultados obtidos, por meio de simulações, que demonstram benefícios na utilização de dados contextuais na tomada de decisão em redes heterogêneas.

Com relação à taxa de transmissão em redes veiculares, o trabalho de [Shankar et al. 2008] propõe a seleção de taxa de transferência baseando-se em informações de contexto para redes veiculares a partir da camada de aplicação. Por meio de uma adaptação, pela qualidade da conexão, é realizada a seleção da taxa de transferência. Em [Campolo and Molinaro 2010] e [Nwizege et al. 2013] os autores avaliam o desempenho e propõem algoritmos de seleção de taxas de transmissão. Em ambos os trabalhos a inferência da taxa de transmissão e realizada por meio dos dados de contexto da distância da posição dos veículos em relação ao outro ponto de comunicação.

Diferentemente dos estudos da literatura, este trabalho considera os contextos individuais e coletivos dos usuários, além da disponibilidade de recursos para atender às demandas da melhor maneira coletiva, sem degradar o consumo de recursos. Para isso diferentes algoritmos são usados intercaladamente de acordo com os contextos atuais.

\section{Descrição da Solução}

A solução proposta baseia-se na metodologia de resolução de conflitos coletivos disponibilizada na literatura por [Silva. 2010]. Foram agregados a esta metodologia as particularidades das redes veiculares e algoritmos específicos para a resolução dos conflitos pertinentes à disputa por estes recursos. Neste modelo de solução, as Unidades de Acostamento $(R S U s)$ atuam na disponibilização da aplicação com a finalidade de distribuição de conteúdo de entretenimento e no controle da distribuição de largura de banda. As Unidades de Bordo $(O B U s)$ disponibilizam os seus dados de contexto e solicitam os recurso de entretenimento às $R S U s$, que por sua vez verificam e atendem as solicitações de acordo com suas disponibilidades. A insuficiência de recursos disponibilizados pode gerar uma insatisfação no usuário não atendido. Esta solução busca, por meio da escolha de algoritmos mais adequados e mediante o contexto atual da rede e dos veículos, balancear o consumo de recursos (como a largura de banda), o tempo consumido para a resolução dos conflitos e a satisfação geral dos usuários.

\subsection{Instância da Metodologia CReMe para Redes Veiculares}

Neste trabalho, foi desenvolvida uma instância da metodologia CReMe com a finalidade de resolver os conflitos de interesses coletivos ocorridos em aplicações de entretenimento para redes veiculares. O controle na distribuição de recursos acontece devido a quantidade limitada da largura de banda disponibilizada pela infraestrutura por meio de um repositório de algoritmos com opções que podem manter o equilíbrio entre a satisfação dos usuários, o tempo de resposta e o consumo dos recursos disponíveis.

Os elementos que compõem o modelo da aplicação são divididos entre os módulos de Solicitação de Conteúdo que é disponibilizado nas $O B U$ s e o da Instância da metodologia CReMe que estará disponível nas RSUs. 
A aplicação veicular atua de acordo com o seguinte modelo conceitual:

- Inserção de solicitações: Cada $O B U$ pode solicitar um conteúdo de entretenimento à $R S U$ que está conectada, durante uma determinada JTS (Janela de Tempo de Solicitações), que são intervalos de tempo nas quais as $R S U s$ aguardam por solicitações.

- Liberação de largura de banda: A $R S U$ analisa as solicitações feitas durante a $J T S$. As solicitações recebidas por uma $R S U$ são devidamente tratadas pela abordagem de resolução de conflitos.

- Execução da tarefa: Uma quantidade de largura de banda é atribuída a cada $O B U$ solicitante, e assim elas estarão autorizadas a obter o conteúdo.

Todo esse processo, à partir da solicitação até a obtenção do conteúdo, é considerado como sendo uma rodada da aplicação. Para a liberação de banda, a $R S U$ depende das demandas imediatas pelo recurso solicitado e da taxa de transferência de dados residual na mesma. O valor que será concedido à $O B U$ resultará, nos casos em que houver conflitos, do processo de resolução de conflitos gerado na disputa pelo recurso. A disponibilidade de largura de banda de cada $R S U$, em um determinado momento, é dada pela sua taxa de transferência de dados, definida pela tecnologia de comunicação utilizada, subtraindo o valor já alocado aos veículos e que ainda estão em uso do serviço.

\subsection{Detecção e Resolução de Conflitos}

Baseado no modelo de aplicação, um conflito ocorre sempre que a soma da quantidade de largura de banda solicitada por todos os veículos, à partir de uma $R S U$ e em uma determinada rodada, superar a quantidade de banda disponível naquele momento. $\mathrm{Ou}$ seja, um conflito coletivo ocorre quando a soma das solicitações ultrapassa a largura de banda disponível. Caso isso ocorra, o módulo de Resolução de Conflitos é acionado. Caso contrário, as solicitações são atendidas e a rodada é encerrada.

O foco da resolução de conflitos é equilibrar o consumo de recursos com a satisfação dos usuários, mantendo transparente para eles a existência do conflito. Os usuários da aplicação almejam receber o conteúdo solicitado antes de chegar ao seu destino final. Sabe-se que os veículos deslocam a partir de um ponto de origem até um ponto de destino e a uma determinada velocidade média. A disponibilização dos recursos requeridos deve ser suficiente, ou estar o mais próximo de conseguir satisfazer os usuários na suas solicitações. Os recursos que não podem ser utilizados indiscriminadamente pelos algoritmos de resolução de conflitos são o tempo e a largura de banda. Assim sendo, caso o esquema de resolução de conflitos leve muito tempo para resolver a disputa, ou seja, o uso excessivo do recurso tempo, uma $O B U$ pode não estar mais interessada no conteúdo solicitado ou ficar fora de alcance da $R S U$. Além disso, o algoritmo de resolução não deve utilizar muitas trocas de mensagens para resolver os conflitos pois sobrecarregaria ainda mais a rede.

Os algoritmos específicos para resolução de conflitos são armazenados em um repositório de algoritmos conforme regras da metodologia CReMe, e são indicados para uso em um dado momento por meio do cálculo da Distância Euclidiana dos meta-dados correspondentes aos algoritmos recuperados a partir dos valores definidos previamente. Para a proposta de aplicação deste trabalho foram utilizados os seguintes algoritmos:

- Negociação: a $R S U$ troca mensagens com as $O B U s$ com o intuito de negociar e, consequentemente, reduzir a demanda por largura de banda; 
- Divisão Igualitária: a $R S U$ divide igualmente entre todas as $O B U s$ solicitantes a sua largura de banda residual.

Uma simples verificação nos procedimentos do Algoritmo de Negociação mostra claramente que ele utiliza um maior tempo e maior consumo dos recursos de rede. Porém, ele tende a proporcionar melhores níveis de satisfação dos usuários, uma vez que irá fornecer a largura de banda necessária para o usuário receber todo o conteúdo negociado. Por outro lado, o algoritmo de Divisão Igualitária atua mais rapidamente que o de Negociação e aloca a mesma fração da largura de banda para todas as $O B U s$ com solicitações em conflito. Porém isso pode resultar para alguns um valor insuficiente, levando à insatisfação. Desta forma, a metodologia CReMe visa utilizar um dos dois algoritmos de acordo com os contextos atuais, visando o equilíbrio entre a satisfação coletiva dos usuários e o consumo de recursos como tempo de resposta e largura de banda.

\section{Análise dos Resultados}

\subsection{Ambiente de simulação}

O ambiente de simulação utilizado para a implementação do modelo proposto foi a ferramenta de simulação chamada SIAFU [Martin and Nurmi 2006]. A simulação deste modelo utiliza o mapa da cidade de Valência na Espanha, e conta com 300 veículos se locomovendo de um ponto inicial a um destino final. Três RSUs são distribuídas na abrangência do mapa com uma capacidade em largura de banda de $60 \mathrm{Mbps}$ cada. Os veículos se locomovem a velocidades que variam entre 5 e 20 metros por segundo $(\mathrm{m} / \mathrm{s})$. Cada simulação transcorre por um período de 12 horas e durante este tempo, os veículos têm uma possibilidade aleatória, gerada por uma distribuição uniforme, de solicitar um conteúdo à aplicação de entretenimento. Este conteúdo é diferenciado pelo seu tamanho em megabytes (MB) que pode variar de 5 a $6 \mathrm{MB}$. Estes valores são gerados também aleatoriamente por uma distribuição uniforme, obtendo assim tamanhos diferentes de conteúdos mas dentro do intervalo estipulado. Foram criados três cenários para a proposta de resolução de conflitos neste modelo e são apresentados na Tabela 1 .

Tabela 1. Abordagens de cenários para avaliação

\begin{tabular}{|l|l|}
\hline Cenário & Descrição \\
\hline \hline Cenário 1 (CReMe) & Solução proposta usando a metodologia CReMe \\
\hline Cenário 2 (Negociação) & Usa somente o algoritmo de negociação \\
\hline Cenário 3 (Igualitário) & Usa somente o algoritmo de divisão igualitária \\
\hline
\end{tabular}

Os Cenários 2 (Negociação) e 3 (Igualitário) foram criados para serem comparados com a avaliação do Cenário 1 (CReMe) que utiliza a metodologia CReMe. No Cenário 2 (Negociação), somente o algoritmo de negociação é utilizado para ser priorizado um maior índice de satisfação coletiva. Já para o Cenário 3 (Igualitário), foi utilizado o algoritmo de divisão igualitária que prioriza o consumo de recursos.

A CReMe realiza a escolha do algoritmo mais indicado neste modelo por meio da observação de dois parâmetros: a quantidade de largura de banda residual na $R S U$ e a velocidade média dos veículos solicitantes. A razão entre a largura de banda disponível $(L)$ e a velocidade média $(V m)$ dos veículos foram utilizados na obtenção de um índice. Este índice foi chamado de índice de vazão $(i Q)$ e é responsável por representar as condições 
em que o ambiente se encontra naquele instante. Ao detectar estas condições, a CReMe escolhe o algoritmo de resolução de conflitos mais indicado para o índice determinado. Conforme os resultados obtidos pelo cálculo do índice $i Q$, pode-se concluir que quanto maior a largura de banda disponível e menor a velocidade dos veículos, maior será a quantidade de dados possíveis de se transmitir em um determinado intervalo de tempo.

\subsection{Análise das Simulações}

A análise sobre os dados apresentados nesta seção tem como objetivo avaliar a natureza dinâmica da metodologia CReMe para resolução de conflitos coletivos em ambientes pervasivos e cientes de contexto. Por meio da utilização de cada estratégia de resolução de conflitos, apresentadas na Tabela 1, foram comparados os resultados para apresentar a estratégia do Cenário 1, que mantém um equilíbrio entre o tempo gasto para a resolução dos conflitos com a satisfação da resolução em detrimento do recurso de rede. Foram capturados para cada rodada com conflitos, os dados das métricas Consumo de rede, Tempo de resposta e Satisfação Coletiva. Em todos os gráficos, são mostrados os valores médios e os respectivos desvios padrão de 33 rodadas de simulação. Os resultados apresentados na Figura 1 mostram os valores das métricas, obtidos na simulação para cada cenário.

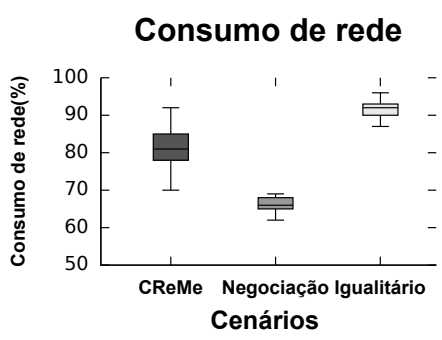

(a) Consumo de rede

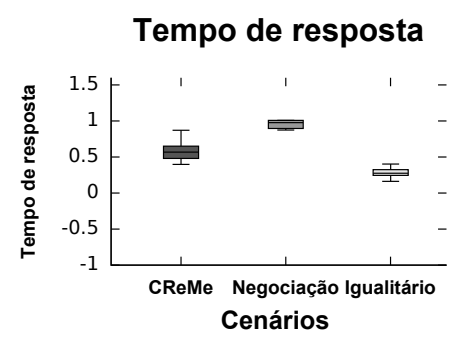

(b) Tempo de resposta

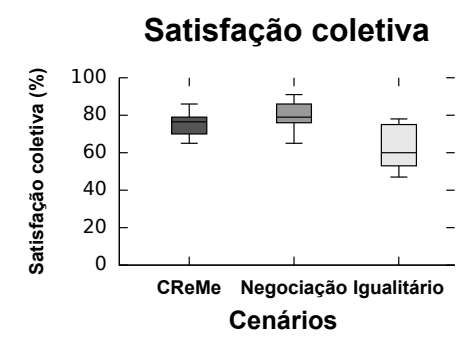

(c) Satisfação coletiva

Figura 1. Métricas - Consumo de rede, satisfação coletiva e tempo de resposta

O consumo de rede é medido rodada a rodada pela porcentagem utilizada do total da largura de banda disponível da RSU requisitada. A Figura 1a apresenta o consumo para os três cenários avaliados. O cenário 1 , que visa o equilíbrio no uso dos recursos, consegue manter o uso controlado da rede sem atingir o seu limite máximo e não deixando o recurso ocioso. Já o cenário 2, no qual é utilizada sempre uma negociação com o veículo, o consumo de largura de banda é menor, porém são feitas negociações com o usuário, para manter a satisfação, utilizando muito do recurso tempo. No cenário 3, o recurso de rede é utilizado indiscriminadamente, deixando-o muitas vezes escasso.

O tempo de resposta é o valor dado ao tempo gasto pelo algoritmo de resolução de conflitos para responder às requisições por largura de banda dos veículos em uma rodada. Este tempo é medido pela diferença entre o instante da requisição e o da resposta. Como se pode observar na Figura 1b, o resultado no cenário 1 situa-se entre o cenário 3 que resolve o conflito em menos tempo e o cenário 2 que consome um tempo maior para proporcionar uma melhor satisfação. Este resultado é esperado, uma vez que, o cenário 1 alterna entre as duas estratégias dos para cada mudança de contexto, visando o equilíbrio entre a satisfação e uso de recursos.

A satisfação coletiva foi coletada com o intuito de verificar o quão satisfeitos ficaram os usuários com a resolução do conflito existente. Foi capturada, a cada rodada, 
a satisfação individual de cada veículo solicitante, e a média destas satisfações resultou na métrica de satisfação coletiva. Na Figura 1c é possível verificar a média de satisfação coletiva dada por cada cenário, sendo que o cenário 2 apresenta uma satisfação coletiva maior, por priorizar sempre esta métrica. No cenário 3, a satisfação é baixa em relação aos outros cenários, mostrando que seu objetivo é de apenas resolver o conflito em um menor tempo. Já o cenário 1 apresenta uma satisfação equilibrada entre os outros dois cenários, pois utiliza a sua estratégia para manter os usuários satisfeitos com um baixo consumo de tempo e consumo controlado de rede.

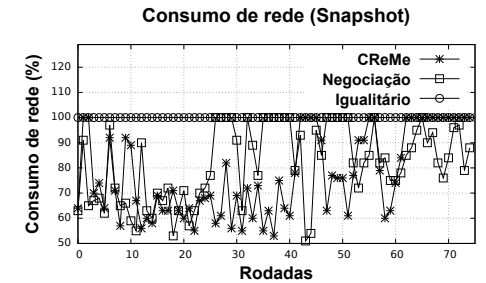

(a) Consumo de rede

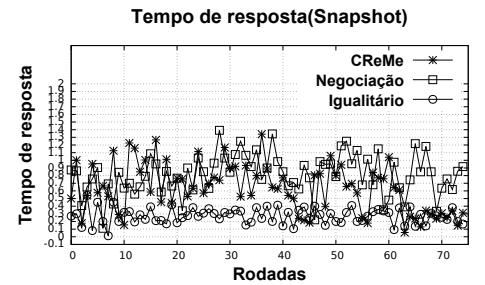

(b) Tempo de resposta

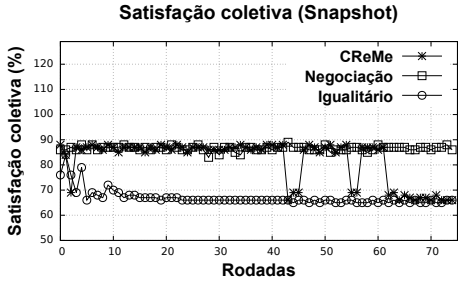

(c) Satisfação coletiva

Figura 2. Snapshots - Consumo de rede, satisfação coletiva e tempo de resposta

Por meio dos snapshots da Figura 2, observa-se o comportamento das métricas avaliadas durante todo o tempo de uma simulação. Na Figura 2a é possível observar comportamentos distintos, de acordo com as características de cada um dos cenários. No Cenário 3, o consumo de recursos alcança sempre o máximo disponível para aquela rodada. Isto ocorre porque o algoritmo utilizado neste cenário divide, igualmente, o total do recurso para todos os solicitantes, conforme a Tabela 1. Já no Cenário 2, o consumo da largura de banda é atenuado por meio da negociação realizada pelo algoritmo para a redução do tamanho do conteúdo e aumento da satisfação. Para o Cenário 1, o consumo de rede oscila entre um valor total, quando é utilizado o algoritmo de divisão igualitária, e valores intermediários, quando o algoritmo indicado para ser utilizado é o de negociação, mostrando assim o dinamismo da CReMe na escolha da estratégia de resolução. Ainda na Figura 2a, é possível observar que, em algumas rodadas sequenciais, o consumo de rede permanece no máximo disponível, mostrando que naquele momento o contexto indica a escolha desta estratégia.

A Figura $2 \mathrm{~b}$ apresenta os valores capturados do tempo de resposta para os três cenários. O Cenário 1 apresenta alguns valores próximos ao do Cenário 2 e outros próximos do Cenário 3, o que mostra a mudança de estratégia da CReMe durante o decorrer da simulação. Os valores apresentados para o Cenário 3 são menores devido o algoritmo utilizado consumir menos tempo de processamento do que o do Cenário 2 que consome tempo para analisar as solicitações, negociar e resolver cada situação. A característica de flexibilidade da CReMe pode ser observada na Figura 2c por meio do comportamento do nível de satisfação do Cenário 1, ao obter satisfações maiores e menores de acordo com a utilização do algoritmo mais indicado para aquela rodada.

\subsection{Análise das simulações com variações de parâmetros}

Análise 1: Alteração na velocidade média dos veículos. Com o objetivo de avaliar e comparar as abordagens de resolução de conflitos em relação à variação da média da velocidade dos veículos, foram utilizados três níveis de velocidades. Os níveis definem 
que os veículos, em média, transitem em uma velocidade próxima ao valor indicado como o mais provável para o mesmo. Os valores foram definidos como $5 \mathrm{~m} / \mathrm{s}$ para o primeiro nível de velocidade "Baixa", $10 \mathrm{~m} / \mathrm{s}$ para o segundo nível definido como "Média"e $20 \mathrm{~m} / \mathrm{s}$ para o terceiro nível definido como "Alta"velocidade.

A satisfação coletiva, obtida e apresentada na Figura 3a, tende a ser reduzida quando a velocidade média está no nível "Alta". Este fato ocorre devido à disponibilidade de tempo de comunicação entre as $O B U s$ e as $R S U s$ se tornar menor. A redução dos valores médios da satisfação ocorre devido ao impacto causado pelo aumento das desistências e não atendimentos quando os veículos estão trafegando em velocidades altas. A Figura $3 b$ superior mostra o aumento do número de desistências em função do aumento da velocidade. Este fato é evidenciado principalmente para a abordagem do Cenário 2, explicando assim a queda na satisfação neste cenário. O aumento no número de desistências é afetado pela combinação do maior tempo de resposta do algoritmo de negociação e o fato dos veículos chegarem ao seu destino mais rapidamente.

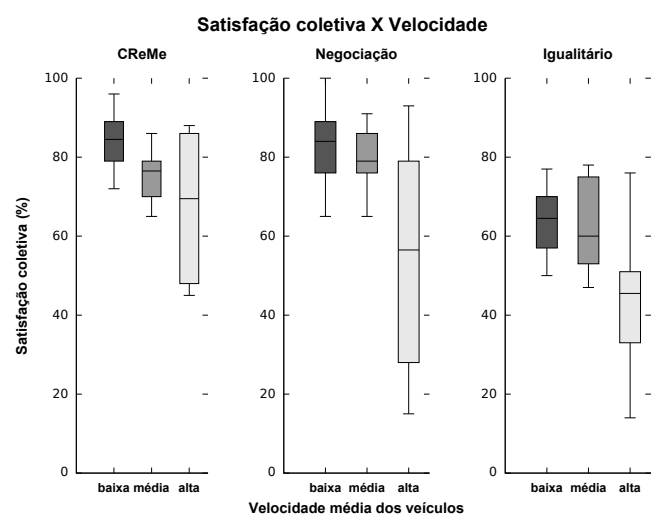

(a) Satisfação coletiva por nível de velocidade

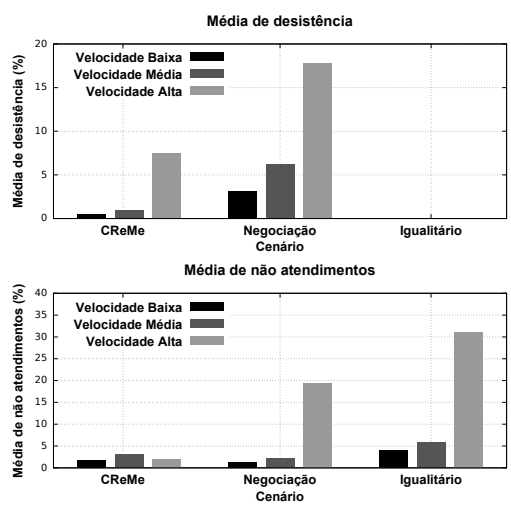

(b) Desistências e não atendimentos

Figura 3. Satisfação coletiva, desistências e não atendimentos em função da alteração na velocidade dos veículos

Para a abordagem de divisão Igualitária no Cenário 3, não há desistência devido ao baixo tempo de resposta, como é apresentado na Figura 3 b com valores zerados. Porém, ocorre um número maior de não atendimentos, mostrado na Figura $3 \mathrm{~b}$ inferior, pelo fato da utilização excessiva de largura de banda por rodada, deixando assim a RSU sobrecarregada para a próxima rodada. Contudo, com velocidades altas, é necessário uma maior vazão de dados para que os veículos consigam receber todo o conteúdo no tempo disponível de conexão.

A abordagem utilizando a metodologia CReMe, o Cenário 1, apresenta um equilíbrio entre as outras duas abordagens, uma vez que utiliza a velocidade como um dos parâmetros para a escolha estratégica do algoritmo de resolução de conflitos. Portanto, com a variação da velocidade entre alta, média ou baixa observa-se como resultado uma satisfação coletiva estável, conforme a Figura 3a. A utilização da metodologia CReMe consegue manter um patamar baixo de desistências mesmo em altas velocidades, como é apresentado na Figura 3b. O fato de acontecer o não atendimento, mostrado na Figura 3b, é atenuado mediante a redução da velocidade dos veículos.

Análise 2: Alteração no tamanho do conteúdo solicitado pelos veículos. Foi re- 
alizada uma comparação do uso da abordagem de resolução de conflitos com diferentes valores para o tamanho do conteúdo solicitado pelos veículos. Os valores possíveis para o tamanho do conteúdo foram medidos em faixas de intervalos definidos como pequeno, médio e grande, sendo os valores de 1 a 1,2 MB para "pequeno", de 5 a $6 \mathrm{MB}$ para "médio", e de 10 a 12 MB para "grande". A satisfação coletiva, apresentada na Figura 4a, foi obtida por meio destas variações e conforme pode ser observado, tende a ser reduzida quando o tamanho do conteúdo aumenta. Isto ocorre devido ao fato que, com um conteúdo maior, é necessário mais recursos para sua utilização.

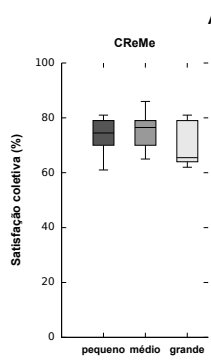

(a) Variação do tamanho de conteúdo

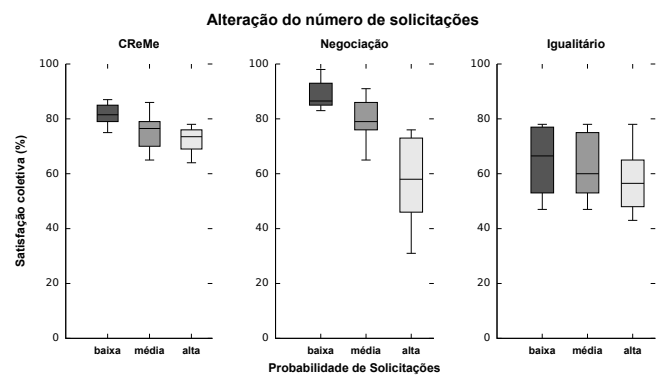

(b) Variação do nível de solicitações

Figura 4. Satisfação coletiva em função da variação de parâmetros

Análise 3: Alteração na probabilidade de solicitações pelos veículos. Esta análise apresenta uma comparação do uso da abordagem de resolução de conflitos com diferentes probabilidades para o veículo solicitar um conteúdo. Para diferenciar as probabilidades de solicitações, foi criado o nível de taxa de solicitação "baixa", na qual uma média de $20 \%$ dos veículos solicitam um conteúdo, o nível "média", em que 50\% dos veículos solicitam conteúdos, e o nível "alta" na qual 100\% dos veículos solicitam conteúdos. O gráfico da Figura $4 \mathrm{~b}$ apresenta, para os três cenários, uma queda na satisfação em função do aumento do número de solicitações. Este resultado mostra que é crítica a escalabilidade em redes veiculares, quando se trata da distribuição de conteúdos de entretenimento. Mais uma vez, o Cenário 1 conseguiu balancear a satisfação dos usuários, mantendo praticamente estáveis esses valores mesmo quando $100 \%$ dos veículos solicitam conteúdos.

\section{Conclusão}

Este trabalho propôs e avaliou uma solução para resolver os conflitos que ocorrem em redes veiculares quando os veículos exigem, simultaneamente, mais largura de banda do que a disponível para a aplicação. Os resultados obtidos mostram que este tipo de conflito deve ser identificado e resolvido de acordo com o contexto, para satisfazer os usuários e manter um consumo eficiente dos recursos. Esta abordagem é baseada em uma metodologia chamada CReMe, que equilibra a satisfação dos usuários com o consumo de recursos. Os resultados revelam a natureza flexível e dinâmica da metodologia CReMe. Os benefícios apresentados pelo comportamento dinâmico e adaptativo da metodologia CReMe são evidentes, na qual são escolhidas as formas de resolução do problema de acordo com as características apresentadas pelo contexto atual da aplicação. Por meio desta solução, foi possível balancear o nível de satisfação dos usuários com um consumo controlado de rede e tempo de resposta. Os próximos passos para este trabalho envolvem a utilização da metodologia CReMe de forma distribuída, fazendo com que os veículos resolvam, por si próprios, os conflitos existentes na disputa pelos recursos. 


\section{Agradecimento}

O presente trabalho foi realizado com o apoio financeiro da CAPES - Brasil e do CNPq (573.738/2008-4 INCT NAMITEC).

\section{Referências}

Campolo, C. and Molinaro, A. (2010). Data rate selection in wbss-based ieee $802.11 \mathrm{p} /$ wave vehicular ad hoc networks. In Communication Systems Networks and Digital Signal Processing (CSNDSP), 2010 7th International Symposium on, pages 412-416.

Costa-Montenegro, E., Quinoy-Garciia, F., Gonzaalez-Castano, F., and Gil-Castineira, F. (2012). Vehicular entertainment systems: Mobile application enhancement in networked infrastructures. Vehicular Technology Magazine, IEEE, 7(3):73-79.

Jiau, M. K., Huang, S. C., Hwang, J. N., and Vasilakos, A. V. (2015). Multimedia services in cloud-based vehicular networks. IEEE Intelligent Transportation Systems Magazine, 7(3):62-79.

Martin, M. and Nurmi, P. (2006). A generic large scale simulator for ubiquitous computing. In Mobile and Ubiquitous Systems - Workshops, 2006. 3rd Annual International Conference on, pages 1-3.

Nwizege, K. S., He, J., Kim, K. S., and Igic, P. (2013). Performance evaluation of adaptive context aware rate selection algorithm (acars) for road safety applications in vehicular network. In Modelling Symposium (EMS), 2013 European, pages 640-646.

Perera, C., Zaslavsky, A., Christen, P., and Georgakopoulos, D. (2014). Context aware computing for the internet of things: A survey. IEEE Communications Surveys Tutorials, 16(1):414-454.

Sarakis, L., Orphanoudakis, T., Leligou, H. C., Voliotis, S., and Voulkidis, A. (2016). Providing entertainment applications in vanet environments. IEEE Wireless Communications, 23(1):30-37.

Sepulcre, M., Gozalvez, J., Altintas, O., and Kremo, H. (2015). Context-aware heterogeneous v2i communications. In Reliable Networks Design and Modeling (RNDM), 2015 7th International Workshop on, pages 295-300.

Shankar, P., Nadeem, T., Rosca, J., and Iftode, L. (2008). Cars: Context-aware rate selection for vehicular networks. In Network Protocols, 2008. ICNP 2008. IEEE International Conference on, pages 1-12.

Silva., T. R. d. M. B. (2010). Tratamento de conflitos coletivos em sistemas ubíquos cientes de contexto. PhD thesis, Universidade Federal de Minas Gerais.

Wan, J., Zhang, D., Zhao, S., Yang, L., and Lloret, J. (2014). Context-aware vehicular cyber-physical systems with cloud support: architecture, challenges, and solutions. Communications Magazine, IEEE, 52(8):106-113.

Yang, Z., Li, M., and Lou, W. (2012). Codeplay: Live multimedia streaming in vanets using symbol-level network coding. Wireless Communications, IEEE Transactions on, 11(8):3006-3013. 